\title{
Core Collapse Supernova Models and Nucleosynthesis
}

\author{
Ken'ichi Nomoto \\ Kavli Institute for the Physics and Mathematics of the Universe (WPI) \\ The University of Tokyo, Kashiwa, Chiba 277-8583, Japan \\ email: nomoto@astron.s.u-tokyo.ac.jp
}

\begin{abstract}
After the Big Bang, production of heavy elements in the early Universe takes place in the first stars and their supernova explosions. The nature of the first supernovae, however, has not been well understood. The signature of nucleosynthesis yields of the first supernovae can be seen in the elemental abundance patterns observed in extremely metal-poor stars. Interestingly, those abundance patterns show some peculiarities relative to the solar abundance pattern, which should provide important clues to understanding the nature of early generations of supernovae. We review the recent results of the nucleosynthesis yields of massive stars. We examine how those yields are affected by some hydrodynamical effects during the supernova explosions, namely, explosion energies from those of hypernovae to faint supernovae, mixing and fallback of processed materials, asphericity, etc. Those parameters in the supernova nucleosynthesis models are constrained from observational data of supernovae and metal-poor stars.
\end{abstract}

Keywords. stellar evolution, supernova, nucleosynthesis

\section{Introduction}

In the Big Bang Universe, the first heavier elements, such as $\mathrm{C}, \mathrm{O}, \mathrm{Ne}, \mathrm{Mg}, \mathrm{Si}$ and Fe, must be synthesized in the evolution and explosion of the first stars (metal-free $=$ Population III = Pop III stars) early in the history of the universe. The massive first stars evolve to explode as the first supernovae ( $\mathrm{SNe}$ ), which release large explosion energies and eject nucleosynthetically-enriched materials.

In the early Universe when the metal content was extremely low, the enrichment by a single supernova $(\mathrm{SN})$ can dominate the pre-existing metal contents. Then the abundance pattern of the enriched gas may reflect nucleosynthesis in the individual SN. The next generation of stars formed from the enriched gas and the long-lived low mass stars may be observed as extremely metal-poor (EMP) stars (Beers \& Christlieb 2005). Thus the abundance patterns of EMP stars can constrain the nucleosynthetic yields of the Pop III SN and thus the mass range of the first stars. Beers \& Christlieb (2005) have defined the metal-poor stars with metallicity $[\mathrm{Fe} / \mathrm{H}] \dagger$ to coin as follows: Very metal-poor (VMP) stars for $-3 \leqslant[\mathrm{Fe} / \mathrm{H}]<-2$, EMP stars for $-4 \leqslant[\mathrm{Fe} / \mathrm{H}]<-3$, Ultra metal-poor (UMP) stars for $-5 \leqslant[\mathrm{Fe} / \mathrm{H}]<-4$, Hyper metal-poor $(\mathrm{HMP})$ stars for $-6 \leqslant[\mathrm{Fe} / \mathrm{H}]<-5$, and Mega metal-poor $(\mathrm{MMP})$ stars for $[\mathrm{Fe} / \mathrm{H}]<-6$.

Actually, recent observations discovered several EMP stars, whose abundance patterns are quite unusual, such as carbon enhanced metal-poor (CEMP) and hyper metal-poor (HMP) stars (e.g., Beers \& Christlieb 2005), being significantly different from previously known nucleosynthesis yields of massive stars. These new observations have raised important challenges to the stellar evolution and nucleosynthesis theory.

$\dagger[\mathrm{A} / \mathrm{B}]=\log _{10}\left(N_{\mathrm{A}} / N_{\mathrm{B}}\right)-\log _{10}\left(N_{\mathrm{A}} / N_{\mathrm{B}}\right) \odot$, where the subscript $\odot$ refers to the solar value and $N_{\mathrm{A}}$ and $N_{\mathrm{B}}$ are the abundances of elements $\mathrm{A}$ and $\mathrm{B}$, respectively. 
Interestingly, there is another challenge to the conventional stellar evolution and supernova models. That is the establishment of the Gamma-Ray Burst (GRB)-Supernova Connection (e.g., Woosley \& Bloom 2006). Four GRB-associated SNe have been confirmed spectroscopically so far. They are all very energetic supernovae, whose kinetic energy $E$ exceeds $10^{52} \mathrm{erg}$, more than 10 times the kinetic energy of normal core-collapse SNe. (We use the explosion energy $E$ for the final kinetic energy of the explosion.) In the present paper, we use the term 'Hypernova $(\mathrm{HN})$ ' to describe such a hyper-energetic supernova with $E_{51}=E / 10^{51} \mathrm{erg} \gtrsim 10$.

Motivated by these challenges, we briefly review the recent results of core-collapse supernova models and their nucleosynthesis. The comparison between such stellar nucleosynthesis yields and the abundance patterns of EMP/UMP/HMP stars can provide a new approach to find out the individual supernova mechanism, especially for Pop III supernovae (Nomoto et al. 2013).

\section{Progenitor's Mass and Explosion Energy}

The fates of Pop III stars depend on the mass to which the initially low-mass stars grow through mass accretion. For various cases of feedback and mass accretion rates, Ohkubo et al. (2009) calculated the evolution of accreting Pop III stars to show that massive stars may form if the mass accretion is not much reduced during the main-sequence evolution (Fig. 1 (left), Ohkubo et al. 2009). It is possible that Pop III stars were even more massive than $\sim 300 M_{\odot}$, if rapid mass accretion continues during the whole main-sequence phase of Pop III stars (Ohkubo et al. 2006). Here the models of stellar evolution, supernova explosions, and nucleosynthesis are described as a function of the main-sequence mass $M$. These models are constrained from the comparison of theoretical supernova light curves and spectra with observations.

As mentioned in the Introduction, the explosion energies of core-collapse supernovae are fundamentally important quantities, and an estimate of $E \sim 1 \times 10^{51}$ ergs has often been used for nucleosynthesis calculations. A good example is SN1987A.
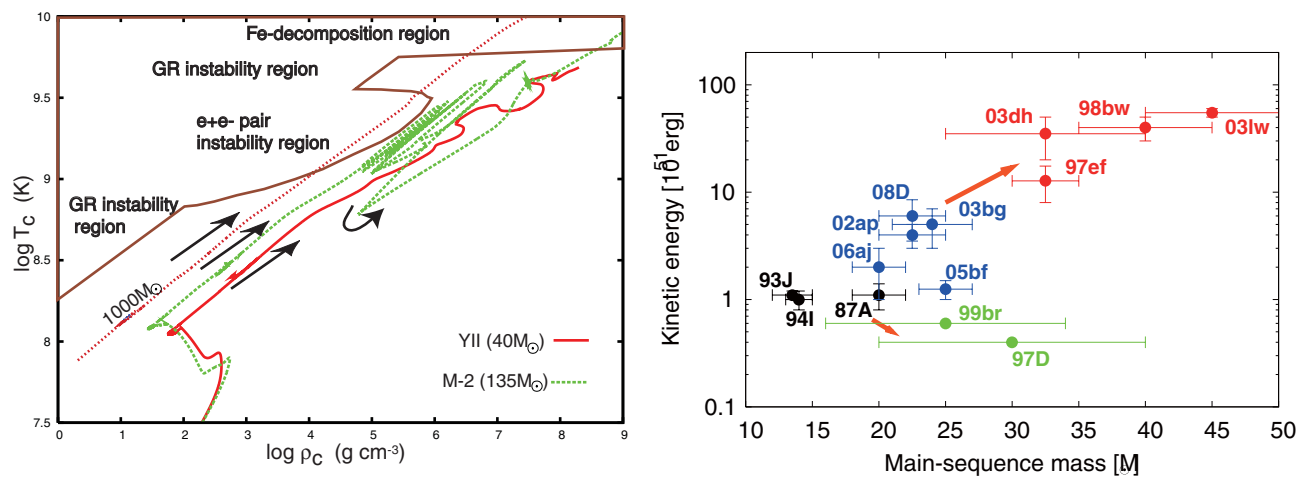

Figure 1. (left:) Evolutionary tracks of the central temperature and central density of Pop III massive stars with mass accretion (Ohkubo et al. 2009). The arrows indicate the direction of the evolution. The numbers in brackets are the final masses for various accretion rates and radiative feedback effects. The evolutionary track without accretion is shown for $M=1000 M_{\odot}$. (right:) The explosion energy as a function of the main-sequence mass of the progenitors for several supernovae/hypernovae. Explosions of $13-25 M_{\odot}$ stars cluster at normal SNe, while explosions of $25-40 M_{\odot}$ stars have a large variety ranging from hypernovae to faint SNe. 
Important change has come from the establishment of the connection between long GRBs and core-collapse SNe from GRB 980425/SN 1998bw, GRB 030329/SN 2003dh, GRB 031203/SN 2003lw, and GRB120422A/SN2012bz (Melandri et al. 2012 and references therein). These GRB-SNe are of Type Ic, showing the broad-line spectra (SNe BL-Ic). The properties of these GRB-SNe, such as the ejected mass (the main-sequence mass of the progenitor) and the kinetic energy of explosion, have been estimated from the comparison between the observed light curve and spectra and their theoretical models (Nomoto et al. 2006). As summarized in Figure 1 (right), these GRB-SNe have similar properties; they are all hypernovae with $E_{51} \sim 30-50$ and synthesize $0.3-0.5 M_{\odot}$ of ${ }^{56} \mathrm{Ni}$. The mass estimates, obtained from fitting the optical light curves and spectra, place hypernovae at the high-mass end of SN progenitors.

An X-Ray Flash-SN connection has also been found: GRB 060218/SN 2006aj and GRB 100316D/SN 2010bh. Compared with the above GRB-SNe, SN 2006aj is less energetic $\left(E_{51} \sim 2\right)$ and its progenitor mass is smaller, $\sim 20 M_{\odot}$, while SN 2010bf may be as energetic as $E_{51} \sim 10$ (Bufano et al. 2012 and references therein).

In contrast, SNe II $1997 \mathrm{D}$ and $1999 \mathrm{br}$ were very faint SNe with very low $E$ (e.g., Turatto et al. 1998). In the $E-M$ diagram (Fig. 1 (right)), therefore, we propose that SNe from stars with $M \gtrsim 20-25 M_{\odot}$ have different $E$, with a bright, energetic "hypernova branch" at one extreme and a faint, low-energy SN branch at the other (Nomoto et al. 2003). For the faint SNe, the explosion energy was so small that most ${ }^{56} \mathrm{Ni}$ fell back onto the compact remnant. Thus the faint SN branch may become even a "failed" SN branch at larger $M$. Between the two branches, there may be a variety of SNe.

This trend might be interpreted as follows. Stars more massive than $\sim 25 M_{\odot}$ form a black hole at the end of their evolution. Stars with non-rotating black holes are likely to collapse "quietly", ejecting a small amount of heavy elements (Faint supernovae). In contrast, stars with rotating black holes are likely to give rise to hypernovae. The hypernova progenitors might form the rapidly rotating cores by spiraling-in of a companion star in a binary system.

\section{8 - $10 M_{\odot}$ Stars and Electron Capture Supernovae}

Stars in the mass range of $\sim 8 M_{\odot}<M<10 M_{\odot}$ can become "electron capture supernovae", if the electron-degenerate $\mathrm{O}-\mathrm{Ne}-\mathrm{Mg}$ core mass grows to $1.38 M_{\odot}$ and the central density reaches $4 \times 10^{9} \mathrm{~g} \mathrm{~cm}^{-3}$ before the whole super-AGB envelope is lost by mass loss. (Thus the lower limit of this mass range depends on the mass loss rate.) At such a high central density, the electron Fermi energy exceeds the threshold for electron captures ${ }^{24} \mathrm{Mg}\left(\mathrm{e}^{-}, \nu\right)^{24} \mathrm{Na}\left(\mathrm{e}^{-}, \nu\right)^{20} \mathrm{Ne}$ and ${ }^{20} \mathrm{Ne}\left(\mathrm{e}^{-}, \nu\right){ }^{20} \mathrm{~F}\left(\mathrm{e}^{-}, \nu\right)^{20} \mathrm{O}$. The resultant decrease in $Y_{e}$ triggers collapse (Nomoto 1987).

The resultant explosion is induced by neutrino heating, and is weak with the kinetic energy as low as $E \sim 10^{50} \mathrm{erg}$ (Kitaura et al. 2006). These stars produce little $\alpha$-elements and Fe-peak elements, but might be another sources of $\mathrm{Zn}$ and light p-nuclei.

Nucleosynthesis in the supernova explosion of a $9 M_{\odot}$ star (Wanajo et al. 2009) shows that the largest overproduction is shared by ${ }^{64} \mathrm{Zn},{ }^{70} \mathrm{Se}$, and ${ }^{78} \mathrm{Kr}$. The ${ }^{64} \mathrm{Zn}$ production provides an upper limit to the occurrence of exploding O-Ne-Mg cores at about $20 \%$ of all core-collapse supernovae. This SN may produce a significant amount of weak $r$-process elements as well as ${ }^{48} \mathrm{Ca}$ from a neutron-rich blob (Wanajo et al. 2013). The ejecta mass of ${ }^{56} \mathrm{Ni}$ is $0.002-0.004 M_{\odot}$, much smaller than the $\sim 0.1 M_{\odot}$ in more massive progenitors. The light curve of electron capture supernova can be consistent with SN 1054 (the Crab Nebula's supernova) (Tominaga, Blinnikov, \& Nomoto 2013). 


\section{10 - $13 M_{\odot}$ Stars and Faint Supernovae}

The stars of $10-13 M_{\odot}$ undergo off-center neon ignition due to the efficient neutrino cooling in the semi-degenerate $\mathrm{O}+\mathrm{Ne}+\mathrm{Mg}$ core (Nomoto \& Hashimoto 1988); this is in contrast to the $13 M_{\odot}$ star where neon is ignited at the center. The neon flame propagates inward due to core contraction. For $10-12 M_{\odot}$, whether or not the neon flame reaches the center needs further study. If the neon flame is quenched, the central region could form an electron-degenerate $\mathrm{O}-\mathrm{Ne}-\mathrm{Mg}$ core surrounded by a layer with the neon-burning products, i.e., a Si-Fe-rich layer. It would become an electron capture supernova as in lower mass stars.

For stars with $M \gtrsim 12 M_{\odot}$, the neon flame reaches the center (Nomoto \& Hashimoto 1988). Then, the core would evolve into an Fe core smaller than $1.4 M_{\odot}$. The explosion of a star with such a small mass Fe core could be powered by neutrino heating, becoming a weak SN as in the electron capture supernovae with O-Ne-Mg cores (Müller et al. 2012). They would eject only a small amount of heavy elements.

The Fe core collapse of these stars would certainly lead to the formation of a neutron star (NS) because there exists a steep density gradient around $\sim 1.4 M_{\odot}$, and the outer envelope is too extended to accrete and further increase the mass of the collapsing core beyond $1.4 M_{\odot}$. The resultant SNe tend to be faint because a negligibly small amount of ${ }^{56} \mathrm{Ni}$ is ejected (Müller et al. 2012). Such an SN may correspond to faint supernovae (Smartt 2009). A possible case of such a faint supernova from this mass range is the Type Ib SN 2005cz, which is unusually faint and rapidly fading (Kawabata et al. 2010).

\section{13 - $25 M_{\odot}$ Stars and Normal Supernovae}

These stars undergo Fe-core collapse to form a NS, and produce significant amount of heavy elements from $\alpha$-elements to Fe-peak elements. The boundary mass between the NS and black hole $(\mathrm{BH})$ formation, $M_{\mathrm{NS} / \mathrm{BH}} \sim 20-25 M_{\odot}$, is only tentative.

For this mass range, SN 1987A in the LMC has provided the most detailed constraints on the explosion model (Arnett et al. 1989). The modeling of nearby supernovae suggest that the stars in the mass range of $13 M_{\odot}-M_{\mathrm{NS} / \mathrm{BH}}$ undergo neutron star-forming $\mathrm{Fe}$ core-collapse and induce normal core collapse supernovae. These SNe produce significant amounts of heavy elements from $\alpha$-elements to Fe-peak elements.

\section{25 - $140 M_{\odot}$ Stars and Hypernovae \& Faint Supernovae}

These stars undergo Fe-core collapse to form a BH. As seen in Figure 1 (right), the resulting black hole-forming SNe seem to be bifurcate into two branches, Hypernovae and Faint SNe. If the BH has little angular momentum, little mass ejection would take place and it would be observed as a Faint SN. On the other hand, a rotating BH could eject matter in the form of jets to make a hypernova. Hypernovae produce a large amount of heavy elements from $\alpha$-elements to Fe-peak elements.

\subsection{Hypernovae}

The right panel of Figure 2 shows the composition of the ejecta for a $25 M_{\odot}$ hypernova model $\left(E_{51}=10\right)$. The nucleosynthesis in a normal $25 M_{\odot} \mathrm{SN}$ model $\left(E_{51}=1\right)$ is also shown for comparison in the left panel of Figure 2 (Umeda \& Nomoto 2002).

We note the following characteristics of nucleosynthesis with very large explosion energies (Nakamura et al. 2001, Nomoto et al. 2001): 
(1) Both the complete and incomplete Si-burning regions shift outward in mass compared with normal supernovae, so that the mass ratio between the complete and incomplete Si-burning regions becomes larger. As a result, higher energy explosions tend to produce larger $[(\mathrm{Zn}, \mathrm{Co}, \mathrm{V}) / \mathrm{Fe}]$ and smaller $[(\mathrm{Mn}, \mathrm{Cr}) / \mathrm{Fe}]$, which can explain the trend observed in very metal-poor stars.

(2) In complete Si-burning of hypernovae, elements produced by $\alpha$-rich freeze-out are enhanced. Hence, elements synthesized through capturing $\alpha$-particles, such as ${ }^{44} \mathrm{Ti},{ }^{48} \mathrm{Cr}$, and ${ }^{64} \mathrm{Ge}$ (decaying into ${ }^{44} \mathrm{Ca},{ }^{48} \mathrm{Ti}$, and ${ }^{64} \mathrm{Zn}$, respectively) are more abundant.

(3) Oxygen burning takes place in more extended regions for larger E. More O, C, and $\mathrm{Al}$ are burned to produce a larger amount of burning products such as $\mathrm{Si}$, S, and Ar. Therefore, hypernova nucleosynthesis is characterized by large abundance ratios of $[\mathrm{Si}, \mathrm{S} / \mathrm{O}]$, which can explain the abundance feature of M82 (Umeda et al. 2002).

\subsection{Faint Supernovae}

In contrast to Hypernovae, faint supernovae undergo extensive fallback of processed materials. The ejecta of fallback supernovae have large $[\mathrm{C} / \mathrm{Fe}]-[\mathrm{Al} / \mathrm{Fe}]$ at low metallicity. These patterns could explain the abundance patterns observed in CEMP stars.

Note that, in spherical explosions, substantial fallback occurs for relatively low $E$. However, in the jet-induced explosions, fallback occurs even for high $E$ explosions. Note also the stellar mass dependence of the abundance pattern is quite weak. In comparing with the observed patterns of CEMP stars, it is difficult to identify the progenitor's mass.

\subsection{Pulsational Instability in Pre-Collapse Stars}

Stars more massive than $M \sim 90 M_{\odot}$ undergo nuclear instabilities and associated pulsations $(\epsilon$-mechanism) at various nuclear burning stages depending on the mass loss and thus metallicity (Heger \& Woosley 2002).

(1) Oxygen Burning: For the $M=137 M_{\odot}$ Pop III star, the evolutionary track for the evolution of the central density and temperature is very close to (but outside of) the " $\mathrm{e}^{-} \mathrm{e}^{+}$pair-instability region" of $\Gamma<4 / 3$, where $\Gamma$ denotes the adiabatic index (Fig. 1 (left)). During oxygen burning, the central temperature and density of such a massive
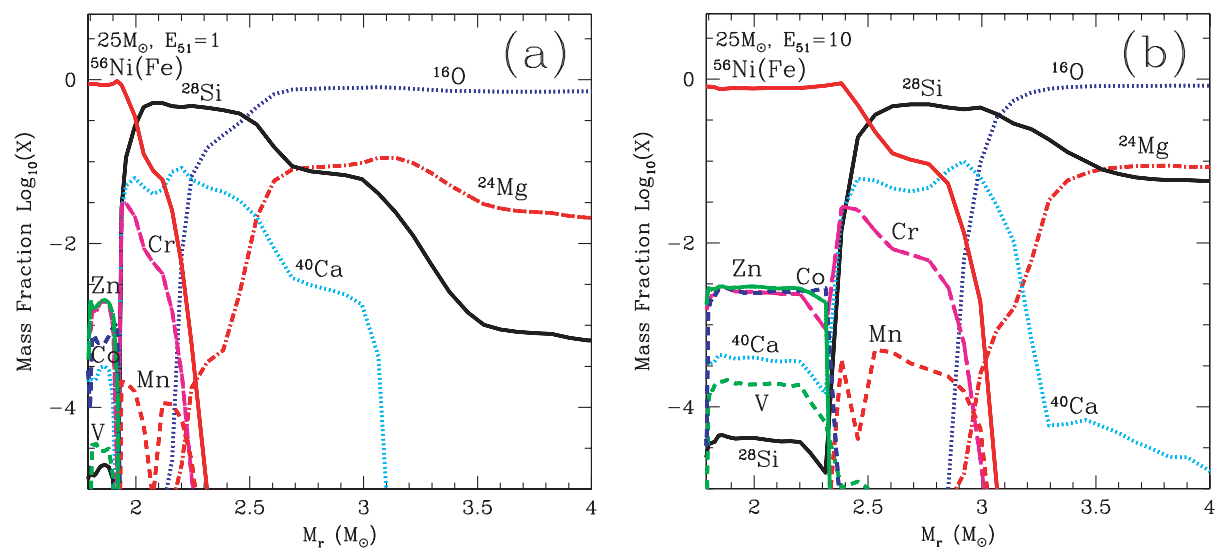

Figure 2. Abundance distribution against the enclosed mass $M_{r}$ after the explosion of Pop III $25 M_{\odot}$ stars with $E_{51}=1$ (a) and $E_{51}=10$ (b) (Umeda \& Nomoto 2002, Tominaga, Umeda, \& Nomoto 2007b). The high explosion energy in hypernovae leads to shift the complete and incomplete Si-burning regions outward in mass and to enhance $\alpha$-rich freeze-out in the complete Si burning layer. 
star oscillate several times (Woosley et al. 2007, Ohkubo et al. 2009). This is because in such massive stars radiation pressure is so dominant that $\Gamma$ is close to $4 / 3$, and thus the inner core of the stars easily expands with the nuclear energy released by O-burning. Once it expands, the temperature drops suddenly, the central O-burning is weakened, and the stellar core in turn shrinks. Since only a small amount of oxygen is burnt for each cycle, these pulsations occur many times. In extreme cases, the pulsation could induce dynamical mass ejection and optical brightening as might be observed in the brightest SN 2006gy (Woosley et al. 2007).

(2) Silicon Burning: $M \sim 90 M_{\odot}$ stars undergo nuclear instability due to silicon burning and pulsates several times (Umeda \& Nomoto 2008). The amplitude of the pulsation due to Si-burning in the central density and temperature is smaller than Oburning (Ohkubo et al. 2009).

(3) Core-Collapse and ${ }^{56}$ Ni Production: Eventually, these $\sim 90-140 M_{\odot}$ stars undergo Fe-core collapse to form BHs. Then hypernova-like energetic SNe could occur to produce large amount ${ }^{56} \mathrm{Ni}$. The synthesized ${ }^{56} \mathrm{Ni}$ mass increases with the increasing $E$ and $M$. For $E=3 \times 10^{52} \mathrm{ergs},{ }^{56} \mathrm{Ni}$ masses of up to $2.2,2.3,5.0$, and $6.6 M_{\odot}$ can be produced for low metallicity $(Z=0.0001)$ progenitors with $M=30,50,80$ and $100 M_{\odot}$ (Umeda \& Nomoto 2008). Thus the upper limit to the mass of ${ }^{56} \mathrm{Ni}$ produced by corecollapse $\mathrm{SNe}\left(M \lesssim 140 M_{\odot}\right)$ would be $\sim 10 M_{\odot}$. The abundance pattern of the ejecta does not depend much on the stellar masses.

Because of the large ejecta mass, the expansion velocities may not be high enough to form broad line features, as has been observed in SN Ic 1999as (Nomoto 2012). Thanks to the large $E$ and ${ }^{56} \mathrm{Ni}$ mass, however, the SNe could be super-luminous supernovae (SLSNe) (Moriya et al. 2010).

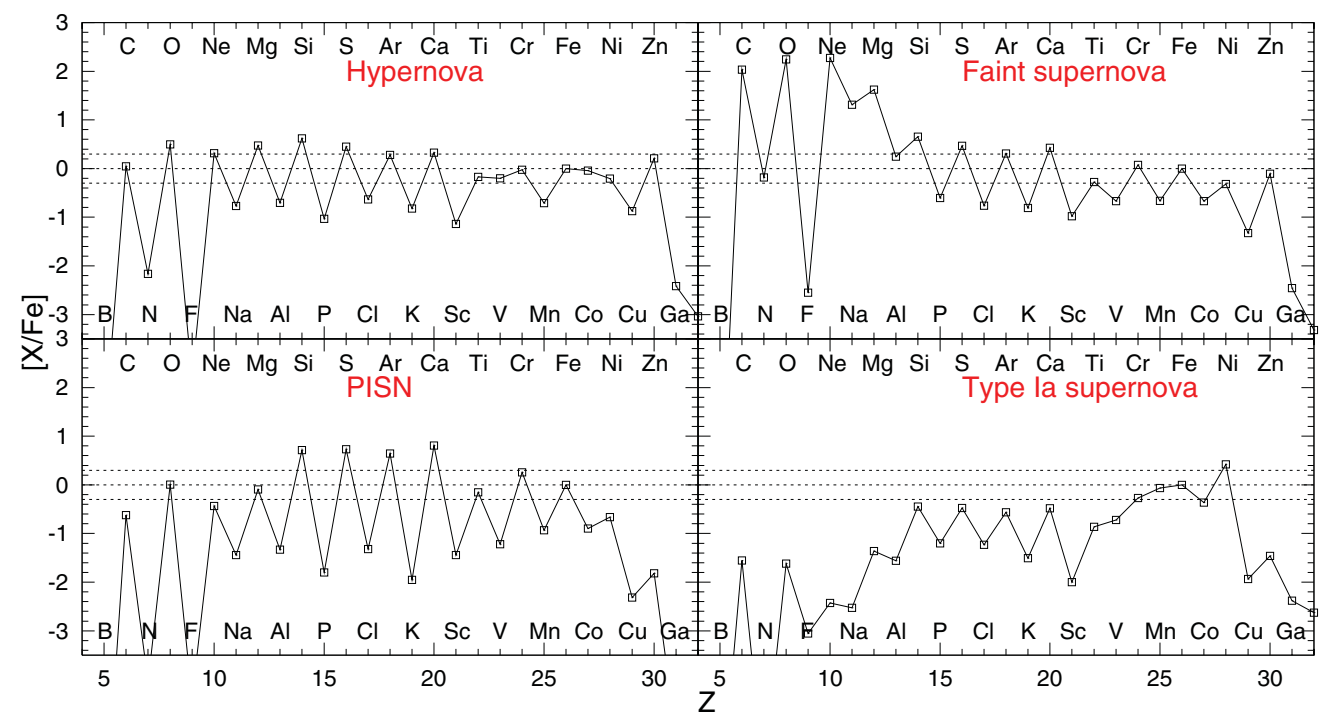

Figure 3. Yields of the core-collapse hypernova (HN), pair-instability supernova (PISN), faint SN, and Type Ia supernova are compared (Nomoto et al. 2013). The faint SN, PISN, and Type Ia supernova are characterized with high $[\mathrm{C} / \mathrm{Fe}]$, low $[\mathrm{Zn} / \mathrm{Fe}]$ and large odd-even effect, and low $[\alpha / \mathrm{Fe}]$, respectively, compared to the hypernova. 


\section{Very Massive Stars}

\subsection{Pair-Instability Supernovae of 140 - $300 M_{\odot}$ Stars}

If very massive stars $\left(M>140 M_{\odot}\right)$ do not lose much mass, they undergo thermonuclear explosions triggered by pair creation instability (pair-instability supernovae: PISN) (Barkat et al. 1967). The star is completely disrupted without forming a BH and thus ejects a large amount of heavy elements, especially ${ }^{56} \mathrm{Ni}$ (e.g., Umeda \& Nomoto 2002, Heger \& Woosley 2002). The largest mass of ${ }^{56} \mathrm{Ni}$ obtained in the PISN models amounts to $\sim 40 M_{\odot}$ (Heger \& Woosley 2002). The resultant radioactive decays of ${ }^{56} \mathrm{Ni}$ and ${ }^{56}$ Co could produce SLSNe.

Figure 3 shows the abundance patters in the ejecta of typical models of the corecollapse hypernova, PISN, faint SN, and Type Ia supernova (SN Ia). The abundance features of PISNe to be compared with the observed abundances are as follows:

- Abundance ratios of iron-peak elements: $[\mathrm{Zn} / \mathrm{Fe}]<-0.8$ and $[\mathrm{Co} / \mathrm{Fe}]<-0.2$. Such small $\mathrm{Zn}$ and Co productions (relative to $\mathrm{Fe}$ ) are due to the low central temperature at the bounce of the collapsing core. This abundance feature of yields is intrinsic to PISNe, because, if the central temperature would become higher, the core collapse would continue due to photodisintegration effects.

- Explosive O-burning leads to large $[(\mathrm{Si}, \mathrm{S}, \mathrm{Ca}) / \mathrm{O}](\sim 0.8)$.

- The odd-even effect is significantly larger in PISNe than core-collapse SNe.

\subsection{Stars with $M \gtrsim 300 M_{\odot}$ and Intermediate Mass Black Holes}

Stars with $300 M_{\odot}<M<3.5 \times 10^{5} M_{\odot}$ enter the pair-instability region but are too massive to be disrupted by PISNe but undergo core collapse, forming intermediate-mass black holes (IMBHs). If such stars formed rapidly rotating black holes, jet-like mass ejection could possibly occur and produce processed material. In fact, for moderately aspherical explosions, the patterns of nucleosynthesis show small $[\mathrm{O} / \mathrm{Fe}]$ and $[\mathrm{Ne} / \mathrm{Fe}]$, and large $[\mathrm{Mg} / \mathrm{Fe}],[\mathrm{Si} / \mathrm{Fe}]$ and $[\mathrm{S} / \mathrm{Fe}]$. Also $[\mathrm{C} / \mathrm{Si}]$ is not so small compared with PISNe. These patterns match the observational data of both the intracluster medium and M82 better than PISNe (Ohkubo et al. 2006).

This result suggests that core-collapse explosions of very massive stars could contribute to chemical enrichment in clusters of galaxies. This might support the view that Pop III core-collapse very massive stars could be responsible for the origin of IMBHs.

Stars more massive than $\sim 3.5 \times 10^{5} M_{\odot}$ (super-massive stars) collapse owing to general relativistic instability before reaching the main-sequence.

\section{Extremely Metal Poor Stars and Abundance Profiling}

\subsection{VMP Stars: Comparison of with Normal Core-Collapse Supernova Yields}

In Figure 4 (left), the averaged abundance pattern of VMP stars $(-2.7<[\mathrm{Fe} / \mathrm{H}]<$ -2.0, Cayrel et al. 2004) are compared with the SN and HN yields integrated over the progenitors of $M=10-50 M_{\odot}$ with the Salpeter's IMF (black solid, Tominaga, Umeda, \& Nomoto 2007b). The observed and theoretical patterns are in reasonable agreement for many elements, although N, K, and Sc are largely underproduced in the model.

\subsection{Extremely Metal-Poor (EMP) Stars}

Figure 4 (right) shows the comparison between the averaged abundances of EMP stars $(-4.2<[\mathrm{Fe} / \mathrm{H}]<-3.5)$ and normal $[\mathrm{C} / \mathrm{Fe}] \sim 0$ (Cayrel et al. 2004)) with the normal Pop III SN yield (blue dashed: $15 M_{\odot}, E_{51}=1$; Tominaga et al. 2007a). It is seen that 
the $\mathrm{SN}$ yield is in reasonable agreement with the observations for $[(\mathrm{Mg}, \mathrm{Al}, \mathrm{Si}) / \mathrm{Fe}]$, but gives too small $[(\mathrm{Mn}, \mathrm{Co}, \mathrm{Zn}) / \mathrm{Fe}]$.

If we compare the observations with the hypernova yield (black solid; $M=20 M_{\odot}$, $\left.E_{51}=10\right)$ in Figure 4 (right), [( $\left.\left.\mathrm{Ti}, \mathrm{Co}, \mathrm{Zn}\right) / \mathrm{Fe}\right]$ are larger and thus in much better agreement with observations than the SN yield. Another example of good agreement between the EMP star (BS 16467-062) and an HN model is seen in Figure 5a (Tominaga, Iwamoto, \& Nomoto 2013).

The difference between the SN and HN models is seen in the abundance distribution of the SN and HN ejecta (Fig.2). Both Co and Zn are synthesized in complete Si burning in a high temperature region, which is more extended in the mass coordinate in the higher $E$ model. Figure 2 also shows that the mass fractions of $\mathrm{Zn}, \mathrm{Co}$, and $\mathrm{V}$ in the complete Si-burning region are larger because of higher entropy in the HN model than the SN model. As a result, the integrated ratios of $\mathrm{Co} / \mathrm{Fe}$ and $\mathrm{Zn} / \mathrm{Fe}$ are larger in higher energy explosions.

\subsection{Carbon-Enhanced Metal-Poor (CEMP) Stars}

A significant fraction of the metal-poor stars show such a large carbon enhancement as $[\mathrm{C} / \mathrm{Fe}] \geqslant+1$, thus being called CEMP stars (e.g., Beers \& Christlieb 2005 and references therein).

The faint SNe occur as a result of fallback of a large amount of radioactive ${ }^{56} \mathrm{Ni}$ whose decay into Fe powers the SN light curve (Fig. 1 (right)). Since the fallback of carbon is less than Fe because of the outer location of $\mathrm{C}$, large $[\mathrm{C} / \mathrm{Fe}]$ results.

Figure 5b shows that the abundance pattern of a CEMP star (CS 29498-043: Aoki et al. 2004) is well reproduced by a single faint $\mathrm{SN}\left(M=25 M_{\odot}\right.$, Tominaga, Iwamoto, \& Nomoto 2013).

The physical mechanism of the mixing-fallback that can yields $[\mathrm{C} / \mathrm{Fe}]>1$ would be the jet-like explosion (Tominaga et al. 2007a) rather than the Rayleigh-Taylor instability resulting in $[\mathrm{C} / \mathrm{Fe}] \lesssim+0.5$.

Most CEMP stars show $[\mathrm{O} / \mathrm{Mg}]>1$. Faint SNe enhance $[\mathrm{O} / \mathrm{Fe}]$ more than $[\mathrm{Mg} / \mathrm{Fe}]$. This is because $\mathrm{Mg}$ is synthesized in the inner region, so that more $\mathrm{Mg}$ falls back onto the central remnant than $\mathrm{O}$.
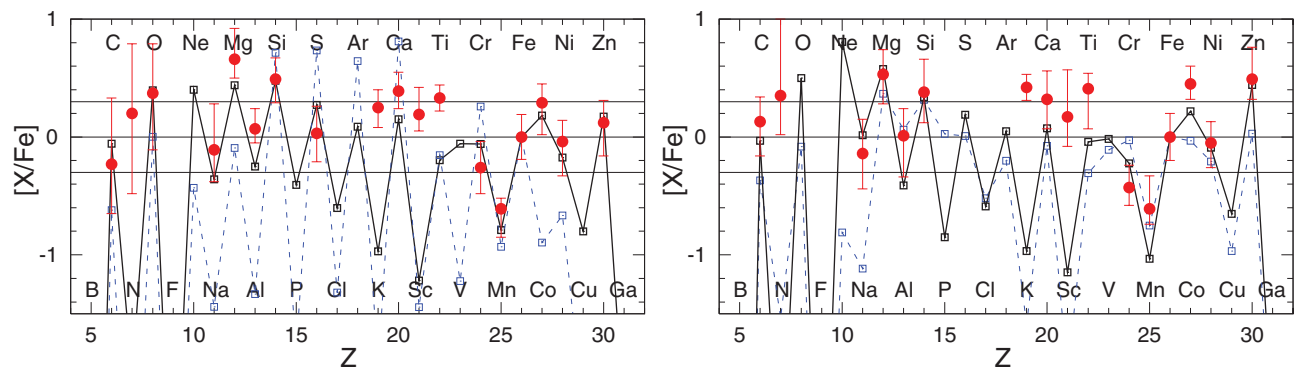

Figure 4. (left:) The abundance pattern of VMP stars with $-2.7<[\mathrm{Fe} / \mathrm{H}]<-2.0$ filled circles with bars is shown to be in good agreement with the IMF integrated yield of Pop III SNe and HNe from $10 M_{\odot}$ to $50 M_{\odot}$ (black solid, Tominaga, Umeda, \& Nomoto 2007b), but not with the $200 M_{\odot}$ PISN yield (blue dashed, Umeda \& Nomoto 2002). (right:) Averaged elemental abundances of stars with $-4.2<[\mathrm{Fe} / \mathrm{H}]<-3.5$ compared with the normal SN yield (blue dashed: $\left.15 M_{\odot}, E_{51}=1\right)$ and the $\mathrm{HN}$ yield $\left(20 M_{\odot}, E_{51}=10\right)$ with the mixing and fallback (black solid). The HN yield have larger $[(\mathrm{Ti}, \mathrm{Co}, \mathrm{Zn}) / \mathrm{Fe}]$ and thus is in better agreement with the EMP stars than the SN yield. 
8.4. Ultra Metal-Poor (UMP) and Hyper Metal-Poor (HMP) Stars

The elemental abundance patterns of these HMP stars provide a key to the answer to the above questions. The abundance patterns of HE1327-2326 (Frebel et al. 2005) and HE0107-5240 (Collet et al. 2006) are quite unusual. The striking similarity of $[\mathrm{Fe} / \mathrm{H}]$ (= $=-5.4$ and -5.2 for HE1327-2326 and HE0107-5240, respectively) and $[\mathrm{C} / \mathrm{Fe}](\sim+4)$ suggests that similar chemical enrichment mechanisms operated in forming these HMP stars. However, the $\mathrm{N} / \mathrm{C}$ and $(\mathrm{Na}, \mathrm{Mg}, \mathrm{Al}) / \mathrm{Fe}$ ratios are more than a factor of 10 larger in HE1327-2326. In order for the theoretical models to be viable, these similarities and differences should be explained self-consistently.

\section{Concluding Remarks}

We report on the properties and nucleosynthesis of the two distinct classes of massive SNe: 1) very energetic Hypernovae, whose kinetic energy is more than 10 times that of normal core-collapse SNe, and 2) very faint and low energy SNe (Faint SNe). These two new classes of SNe are likely to be "black-hole-forming" SNe with rotating or nonrotating black holes. Nucleosynthesis in Hypernovae is characterized by larger abundance ratios $(\mathrm{Zn}, \mathrm{Co}, \mathrm{V}, \mathrm{Ti}) / \mathrm{Fe}$ than normal $\mathrm{SNe}$, which can explain the observed ratios in EMP stars. Nucleosynthesis in Faint SNe is characterized by a large amount of fall-back, which explains the abundance pattern of the most Fe-poor stars.

These comparisons suggest that black-hole-forming SNe made important contributions to the early Galactic (and cosmic) chemical evolution. We discuss how nucleosynthetic properties resulted from such unusual supernovae are connected with the unusual

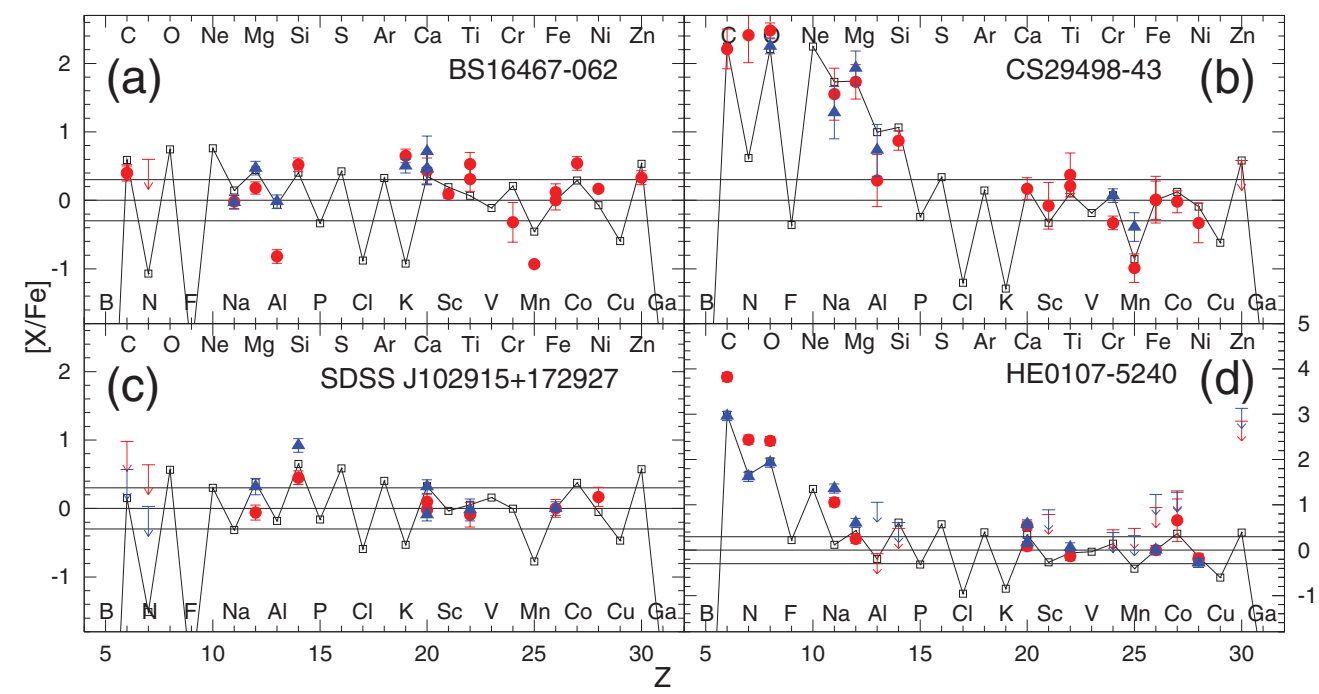

Figure 5. Comparison of the observed abundance patterns (1D-LTE: red filled circles with bars and 3D-(N)LTE: blue filled triangles with bars if available) of EMP (BS 16467-062, Cayrel et al. 2004), CEMP (CS 29498-43, Aoki et al. 2004), UMP (SDSS J102915+172927, Caffau et al. 2011), and HMP stars (HE 0107-5240, Collet et al. 2006) with relevant SN models (Tominaga, Iwamoto, \& Nomoto 2013). The SN models are constructed for the $25 M_{\odot}$ progenitor (Iwamoto et al. 2005). The explosion energies and ejected ${ }^{56} \mathrm{Ni}$ masses of SN models are $E_{51}=20$ and $M\left({ }^{56} \mathrm{Ni}\right)=0.044 M_{\odot}($ EMP star $), E_{51}=20$ and $M\left({ }^{56} \mathrm{Ni}\right)=9.1 \times 10^{-4} M_{\odot}($ CEMP star $)$, $E_{51}=20$ and $M\left({ }^{56} \mathrm{Ni}\right)=1.2 \times 10^{-1} M_{\odot}($ UMP star $)$, and $E_{51}=5$ and $M\left({ }^{56} \mathrm{Ni}\right)=8.0 \times 10^{-5} M_{\odot}$ (HMP star). 
abundance patterns of extremely metal-poor stars. Such connections may provide important constraints on the properties of first stars.

This research has been supported by World Premier International Research Center Initiative, MEXT, Japan.

\section{References}

Aoki, W., Norris, J. E., \& Ryan, S. G., et al. 2004, ApJ, 608, 971

Arnett, W. D., Bahcall, J. N., Kirshner, R. P., \& Woosley, S. E. 1989, ARAA, 27, 629

Barkat, Z., Rakavy, G., \& Sack, N. 1967, Phys. Rev. Letters, 18, 379

Beers, T. C. \& Christlieb, N. 2005, ARAA, 43, 531

Bufano, F., et al. 2012, ApJ, 753, 67

Caffau, E., et al. 2011, Nature, 477, 67

Cayrel, R., et al. 2004, A\&BA, 416, 1117

Christlieb, N., et al. 2002, Nature, 419, 904

Collet, R., Asplund, M., \& Trampedach, R. 2006, ApJ, 644, L121

Frebel, A. et al. 2005, Nature, 434, 871

Heger, A. \& Woosley, S. E. 2002, ApJ, 567, 532

Iwamoto, N., Umeda, H., Tominaga, N., Nomoto, K., \& Maeda, K., 2005, Science, 309, 451

Kawabata, K., Maeda, K., Nomoto, K., et al. 2010, Nature, 465, 326

Kitaura, F. S., Janka, H.-Th., \& Hillebrandt, W. 2006, A\&3A, 450, 345

Melandri, A., et al. 2012, A\&\&A, 547, 82

Moriya, T., Tominaga, N., Tanaka, M., Maeda, K., \& Nomoto, K. 2010, ApJ, 717, 83

Müller, B., Janka, T., \& Heger, A. 2012, ApJ, 761, 72

Nakamura, T., Umeda, H., Iwamoto, K., Nomoto, K., et al. 2001, ApJ, 555, 880

Nomoto, K. 1987, ApJ, 322, 206

Nomoto, K. \& Hashimoto, M. 1988, Phys. Rep., 163, 13

Nomoto, K., Mazzali, P. A., Nakamura, T., et al. 2001, in Supernovae and Gamma Ray Bursts, eds. M. Livio et al. (Cambridge Univ. Press) 144 (astro-ph/0003077)

Nomoto, K. et al. 2003, in IAU Symp. 212, A Massive Star Odyssey, from Main Sequence to Supernova, eds. V. D. Hucht et al.(San Francisco: ASP) 395 (astro-ph/0209064)

Nomoto, K., Tominaga, N., Umeda, H., Kobayashi, C., \& Maeda, K. 2006, Nuclear Phys., A777, 424

Nomoto, K. 2012, in IAU Symp. 279, Death of Massive Stars: Supernovae and Gamma-Ray Bursts, ed. N. Kawai et al. (Cambridge: Cambridge Univ. Press) 1

Nomoto, K., Kobayashi, C., \& Tominaga, N. 2013, ARAA, in press

Ohkubo, T., Umeda, H., Maeda, K., Nomoto, K., Suzuki, T., Tsuruta, S., \& Rees, M. J. 2006, ApJ, 645, 1352

Ohkubo, T., Nomoto, K., Umeda, H., Yoshida, N., \& Tsuruta, S., 2009, ApJ, 706, 1184

Smartt, S. J. 2009, ARAA, 47, 63

Tominaga, N., Maeda, K., Umeda, H., Nomoto, K., Tanaka, M., Iwamoto, N., Suzuki, T., \& Mazzali, P. A. 2007a, ApJ, 657, L77

Tominaga, N., Umeda, H., \& Nomoto, K. 2007b, ApJ, 660, 516

Tominaga, N., Blinnikov, S., \& Nomoto, K. 2013, ApJ, in press

Tominaga, N., Iwamoto, N., \& Nomoto, K. 2013, ApJ, submitted

Turatto, M., Mazzali, P. A., Young, T., Nomoto, K., et al. 1998, ApJ, 498, L129

Umeda, H. \& Nomoto, K. 2002, ApJ, 565, 385

Umeda, H., Nomoto, K., Tsuru, T., \& Matsumoto, H. 2002, ApJ, 578, 855

Umeda, H. \& Nomoto, K. 2003, Nature, 422, 871

Umeda, H. \& Nomoto, K. 2008, ApJ, 673, 1014

Wanajo, S., Nomoto, K., Janka, H.-T., Kitaura, F. S., \& Müller, B. 2009, ApJ, 695, 208

Wanajo, S., Janka, H.-T., \& Müller, B. 2013, ApJ (Letters), 767, L26

Woosley, S. E. \& Bloom, J. S. 2006, ARAA, 44, 507

Woosley, S. E., Blinnikov, S., \& Heger, A. 2007, Nature, 450, 390 